\title{
Manejo y monitoreo anestésico en cirugía de laringectomioía total
}

\author{
Durán Chávez PD. ${ }^{1,2}$, Pacheco A. ${ }^{1}$ \\ 1 Hospital Zenon Santillan, Tucuman, Argentina. \\ 2 Hospital Regional Ramón Carrillo, Santiago del Estero, Argentina.
}

Introducción: El cáncer de laringe es una enfermedad causada por el crecimiento incontrolado de las células de la laringe. Estas patologías afectan 3: 1 hombres, los factores de riesgo son: tabaquismo, alcohol, mayores de 55 años de edad, dieta inadecuada pobre en frutas y verduras. En caso de que este cáncer afecte la zona supraglotica, los síntomas se manifiestan más tarde: disfonía, adenopatías en el cuello, disfagia, disnea, odinofagia. Tratamiento quimioterapia, radioterapia y/o quirúrgica como la laringectomia total (LT).

Descripción del caso: Paciente masculino de 85 años de edad tabaquista de jerarquía, consulta, por disfonía, presenta lesión de cuerdas vocales, se realizo biopsia con informe de carcinoma epidermoide, a la tac se observa lesión que invade cartílago tiroides correspondiente a T4 de estatificación, plan quirúrgico laringectomia total. En quirófano, parámetros deingreso: TA 120/80 mmHg; FC 80 latidos/min; S02 98\%, venoclisis: MSI con abbocath 16G, MSD abbocath $\mathrm{N}^{\circ} 14 \mathrm{G}$, cateterización: de arteria radial izquierda para monitoreo invasivo de TA con abbocath $\mathrm{N}^{\circ}$ 20G. Monitoreo continuo intraoperatorio con CONOX y EV1.000. Premedicación: diclofenac $75 \mathrm{mg}$, ranitidina 50 $\mathrm{mg}$, dexametasona $8 \mathrm{mg}$, metoclopramida $10 \mathrm{mg}$. Cefazolina $2 \mathrm{~g}$. Inducción: fentanilo $100 \mathrm{mcg}$, remifentanilo 0,5 $\mathrm{mcg} / \mathrm{kg} / \mathrm{min}$ propofol $3 \mathrm{mg} / \mathrm{kg} / \mathrm{h}$ atracurio $30 \mathrm{mg}$. IOT satisfactoria con TET espiralado $\mathrm{N}^{\circ} 7 \mathrm{~mm}$ con videolaringo King visión conectado a ARM modo VCV, FIO2 0,60, VT 500 ml, FR 13 resp/min, PEEP 6 cmH2O. Mantenimiento: TIVA con propofol $(1,5 \mathrm{mg} / \mathrm{kg} / \mathrm{h})$, remifentanilo $(0,40-0,80 \mathrm{mcg} / \mathrm{kg} / \mathrm{min})$, ketamina $(0,10-0,12 \mathrm{mcg} / \mathrm{kg} / \mathrm{h})$, lidocaina $(1-1,5 \mathrm{mg} / \mathrm{kg} / \mathrm{h})$ y atracurio $(5 \mathrm{mg}$ refuerzo). Noradrenalina $(0,03-0,05 \mathrm{mcg} / \mathrm{kg} / \mathrm{min})$. Líquidos EV: $700 \mathrm{ml}$ solución fisiológica, $500 \mathrm{ml}$ ringer lactato. Duración de cirugía: 2 horas. Extubación satisfactoria. Analgesia: morfina ev (4 $\mathrm{mg}$ ). Control postquirúrgico: A las $4 \mathrm{~h}$ lúcido, hemodinamicamente estable, sin requerimiento de vasopresores , Ta 150/65 fc 70x' sat 97\% con ventilación espontanea a FiO2 0,21. Drenaje debito serohemático escaso, SNG debito positivo. Diuresis + espontanea. Sin dolor según escala EVA.

Discusión: Los tumores glóticos en estadío temprano pueden ser tratados efectivamente con cirugía o radioterapia, para tumores T1 y T2 incluyen resección de cuerda vocal, microcirugía láser, hemilaringectomía y laringectomía subtotal. La laringectomía total en general se reserva para el cáncer laríngeo más avanzado (T3 y T4) o para pacientes con mala respuesta a tratamientos previos.

Conclusión: La LT es un tratamiento eficaz para el manejo de los pacientes con cáncer de laringe localmente avanzado. Los protocolos de preservación de órgano deberían alcanzar resultados oncológicos similares a los demostrados por la LT.

https://doi.org/10.25237/congresoclasa2019.71 